\title{
STEADY PRANDTL-BATCHELOR FLOWS PAST A CIRCULAR CYLINDER
}

\author{
G. C. HOCKING ${ }^{1}$
}

(Received 1 March, 2006; revised 24 August, 2006)

\begin{abstract}
The high Reynolds number flow past a circular cylinder with a trailing wake region is considered when the wake region is bounded and contains uniform vorticity. The formulation allows only for a single vortex pair trapped behind the cylinder, but calculates solutions over a range of values of vorticity. The separation point and length of the region are determined as outputs. It was found that using this numerical method there is an upper bound on the vorticity for which solutions can be calculated for a given arclength of the cavity. In some cases with shorter cavities, the limiting solutions coincide with the formation of a stagnation point in the outer flow at both separation from the cylinder and reattachment at the end of the cavity.
\end{abstract}

2000 Mathematics subject classification: primary 76B07, 76B10, 76B47.

Keywords and phrases: Prandtl-Batchelor vortex, trailing vortices, wakes, free-streamline theory, integral equations.

\section{Introduction}

The wake behind obstacles is a complicated structure that has been the subject of many studies. Perhaps the simplest model is one in which the pressure in the wake region is assumed to be constant. Many such solutions, computed either numerically, using free streamline theory, or using thin/slender body approximations, are known, see for example $[3,5,10,13,14,18]$. Many of these solutions involve an unbounded wake region, and for flow past a cylinder these are invalid for separation angles less than approximately 55 degrees (because the streamline passes through the body) and for angles greater than approximately 124 degrees (because the two separating streamlines cross), see for example [10, 19]. However, if cusped cavities are assumed $([13,19])$ these restrictions no longer apply and physically real solutions can be obtained for

\footnotetext{
'Mathematics \& Statistics, Murdoch University, Murdoch, WA 6150, Australia; e-mail:

G.Hocking@murdoch.edu.au.

(C) Australian Mathematical Society 2006, Serial-fee code 1446-181 1/06
} 
angles outside of this range. These constant pressure wake solutions provide an interesting starting point for consideration of such flows, but do not generally agree well with experiments.

There have been several attempts to model this flow taking into account viscosity by solving the Navier-Stokes equations numerically, but the numerical method becomes unstable as the Reynolds number increases. For example, $[8,9]$ found this to occur at around $\operatorname{Re}=600$ and then at $\operatorname{Re}=800$.

Batchelor [1, 2] proposed a model for these flows at high Reynolds number that included a region of trapped vorticity surrounded by a flowing irrotational fluid. These Prandtl-Batchelor flows, as they have become known, are probably not stable and are unlikely to be observed in the laboratory, but have been a subject of much study. Few solutions of exactly this type have been computed due to their highly nonlinear character as the location of the dividing streamline is unknown, but some numerical and approximate solutions do exist. Childress [4] performed calculations assuming a slender eddy for flow over a wedge and down a step, while Sadovskii [16] (see also Lamb [11]) showed that vortices can be self-sustaining in an inviscid free stream. Smith [17] extended this by matching these solutions to the flow past a cylinder to create a trailing vortex flow. Moore et al. [15] examined the suggestion of [8] that the Sadovskii vortex is an approximation to the wake behind a circular cylinder and found the comparison encouraging. Vanden-Broeck and Tuck [20] computed flows with an eddy in contact with a concave boundary including corners of various angles. Elcrat et al. [6] used transformations in complex variables and finite differences to compute an exotic array of vortex flows including a circular cylinder. No conditions were applied on the dividing streamline except that it was a streamline.

In this paper, the flow around a cylinder is computed numerically when a region of uniform vorticity is trapped on the downstream side, see Figure 1. The solutions are restricted to those in which there is a single vortex pair and separation from the body and closure of the wake region are smooth. No extra conditions are applied to determine these points. Boundary integral methods are used to compute both the inner and outer flows, one satisfying Laplace's equation and the other a Poisson equation incorporating a uniform vorticity, $\omega$. These flows are then matched at their common boundary according to the appropriate flow conditions.

It is found that for each arclength of the vortex region boundary there is a maximum vorticity at which a solution can be computed using this method, and that as the arclength decreases, the maximal vorticity increases. No solutions with "leading edge" separation were obtained.

In Section 2 we formulate the numerical method for solving the flow problem using an iterative technique. Results and conclusions follow. 


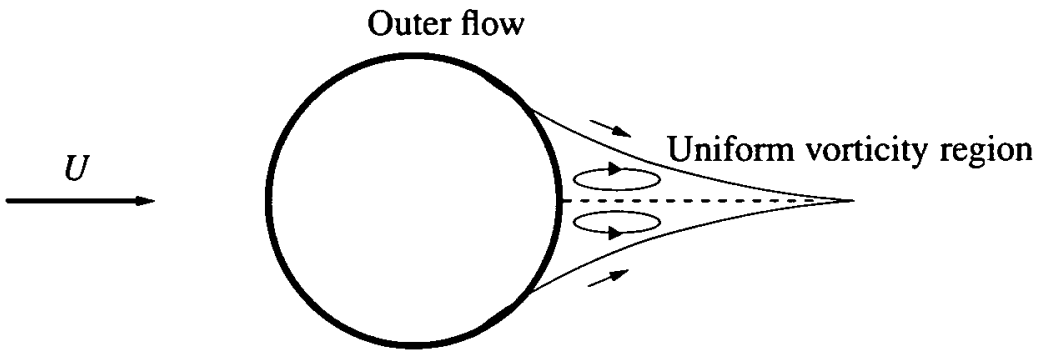

FIGURE 1. Sketch of the flow of a uniform stream past a cylinder with a trailing region of trapped vorticity.

\section{Problem formulation}

The steady motion of an inviscid, incompressible fluid past a circular cylinder behind which there is a finite wake containing a region of uniform vorticity is to be considered. The full problem will consist of solving simultaneously for the flow over the cylinder and wake region and the flow within the wake, where the surface of the wake region is determined as part of the solution. In order to do this we take a two-stage approach in which we "guess" the shape of the wake surface, solve for the flow past this "object" and the flow inside it, and then iterate until the surface shape satisfies the appropriate conditions. In this section we consider the solution for these two flow regions and then the conditions at the vortex sheet separating them.

2.1. Flow past the body and wake The number of parameters in the problem is reduced if we non-dimensionalise with respect to the cylinder radius, $R$, as the length scale, and the free stream (far field) velocity, $U$. In these coordinates, the cylinder radius and free-stream velocity will both have unit value. Using non-dimensional coordinates, let $z=x+i y$ be the physical plane, with the origin in the middle of the cylinder, as shown in Figure 2.

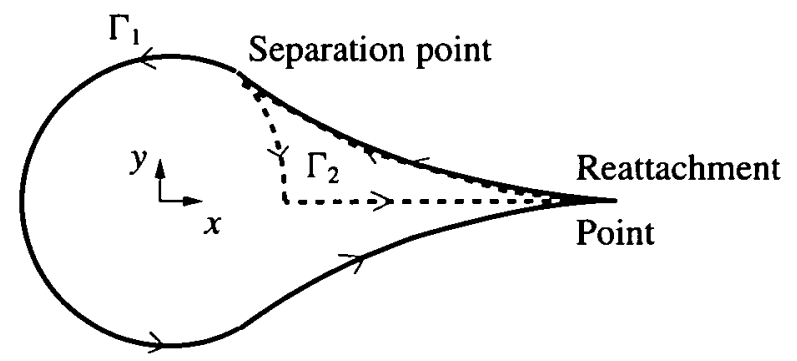

FIGURE 2. Contours of integration, $\Gamma_{1}$ for the outer flow, $\Gamma_{2}$ for the inner, vortex flow.

Let $y= \pm \eta(x),-1<x<x_{L}$, where $x_{L}$ is the location of the reattachment of 
the trailing wake, be the equation of the upper and lower surfaces of the cylinder and the attached dividing streamline. The location of the body surface is known, but the shape of the dividing streamline must be determined as part of the solution. Assuming irrotational flow of an incompressible, inviscid fluid, the velocity potential, $\Phi_{\mathrm{O}}(x, y)$, of the flow field outside of the wake region must satisfy Laplace's equation, that is,

$$
\nabla^{2} \Phi_{\mathrm{O}}(x, y)=0
$$

We solve this problem numerically using a variation on the standard boundary element method (see for example $[7,12]$ ), by noting that the derivative of the complex potential $f^{\prime}(z)=u-i v$ must be an analytic function, where $u$ and $v$ are the velocities in the $x$ and $y$ directions respectively. We define a new function, $\chi(z)$, where $f^{\prime}(z)=1+\chi(z)$, so that $\chi(z) \rightarrow 0$, as $|z| \rightarrow \infty$.

The function $\chi(z)$ must be analytic in the region outside of the cylinder and trailing wake, and we can use Cauchy's integral formula to derive an integral equation that when combined with the other conditions will provide a complete formulation of the problem. Following [7], we apply Cauchy's integral formula to the analytic function $\chi(z)$ around the contour consisting of a circle of radius $\mathscr{R} \rightarrow \infty$ and $\Gamma_{1}$ (see Figure 2). However, since $\chi(z) \rightarrow 0$ as $|z| \rightarrow \infty$, we only need to integrate around the contour $\Gamma_{1}$, giving the equation

$$
i \pi \chi\left(z_{0}\right)=\int_{\Gamma_{1}} \frac{\chi(z)}{z-z_{0}} d z
$$

where $z_{0}$ lies on the boundary.

Let $s$ be the arclength along the interface starting from the leading stagnation point, so that

$$
\left(\frac{d x}{d s}\right)^{2}+\left(\frac{d \eta}{d s}\right)^{2}=1
$$

Using $t$ as the variable of integration and applying the chain rule we obtain

$$
\pi i \chi(z(s))=\int_{0}^{s_{L}} \frac{\chi(z(t)) \frac{d z}{d t}}{z(t)-z(s)} d t-\int_{0}^{s_{L}} \frac{\chi\left(z\left(t_{I}\right)\right) \frac{d z}{d t_{I}}}{z\left(t_{I}\right)-z(s)} d t_{l},
$$

where the subscript $I$ refers to the "image" surface at $y=-\eta(x)$, and $s_{L}$ is the total arclength of the cylinder and trailing wake from front to back.

On the surface of the body and wake region, $u$ and $v$ can be related to each other by noting that the flow must be along the surface, that is,

$$
v(s)=u(s)\left(\frac{d \eta / d s}{d x / d s}\right), \quad 0<s<s_{L},
$$


so that (2.2) represents an integral equation for $u(s)$. Taking the imaginary part, and utilising the symmetry of the situation about the line $y=0$, that is, $x\left(t_{l}\right)=x(t)$, $y\left(t_{l}\right)=-y(t), x^{\prime}\left(t_{l}\right)=x^{\prime}(t), y^{\prime}\left(t_{l}\right)=-y^{\prime}(t), u\left(t_{l}\right)=u(t)$, and $v\left(t_{I}\right)=-v(t)$, and noting $y(s)=\eta(s)$, the integral equation becomes

$$
\pi(u(s)-1)=\int_{0}^{s_{L}}\left([u(t)-1]\left[K_{1}+K_{2}\right]-v(t)\left[K_{3}+K_{4}\right]\right) d t,
$$

where

$$
\begin{aligned}
& K_{1}(x, y: s, t)=\frac{y^{\prime}(t) \Delta x-x^{\prime}(t) \Delta y}{\Delta x^{2}+\Delta y^{2}}, \quad K_{2}(x, y: s, t)=\frac{y^{\prime}(t) \Delta x-x^{\prime}(t) \Delta y_{+}}{\Delta x^{2}+\Delta y_{+}^{2}}, \\
& K_{3}(x, y: s, t)=\frac{x^{\prime}(t) \Delta x+y^{\prime}(t) \Delta y}{\Delta x^{2}+\Delta y^{2}}, \quad K_{4}(x, y: s, t)=\frac{x^{\prime}(t) \Delta x+y^{\prime}(t) \Delta y_{+}}{\Delta x^{2}+\Delta y_{+}{ }^{2}},
\end{aligned}
$$

$\Delta x=x(t)-x(s), \Delta y=y(t)-y(s)$ and $\Delta y_{+}=y(t)+y(s)$. The terms involving $K_{1}$ and $K_{3}$ must be treated as Cauchy-principal-value integrals as there is a simple pole as $t \rightarrow s$ in the integrand. This integral equation, in spite of its rather complex appearance, can be solved numerically to obtain the velocity everywhere on the surface of the object and trailing wake. In order to do this, it is necessary to remove the singularity as $s \rightarrow t$ in the integrand, and so this integral is modified by adding and subtracting a term, giving

$$
\begin{aligned}
u(s)= & E(s)+\frac{1}{\pi} \int_{0}^{s_{L}}\left([u(t)-u(s)] K_{1}+[u(t)-1] K_{2}\right. \\
& \left.-[v(t)-v(s)] K_{3}-v(t) K_{4}\right) d t
\end{aligned}
$$

where

$$
E(s)=1+\frac{1}{\pi}\left([u(s)-1] \arctan \left[\frac{\Delta y}{\Delta x}\right]_{0}^{s_{L}}-\frac{1}{2} v(s) \log \left[\Delta x^{2}+\Delta y^{2}\right]_{0}^{s_{L}}\right) .
$$

The modified integrand now approaches $u^{\prime}(s)$ for the horizontal velocity term (involving $K_{1}$ ) and zero for the vertical velocity term (involving $K_{3}$ ) as $t \rightarrow s$, and is therefore well behaved.

This equation can be solved by taking a discrete representation that gives a linear system of algebraic equations for $u(s)$ if $x(s), y(s)$ are known.

2.2. Flow within the wake The flow in the wake region is still incompressible, but we now assume that there exists a uniform vorticity, $\omega$. Thus the flow can be obtained by solving for the streamfunction, $\psi$, that satisfies a Poisson equation

$$
\nabla^{2} \psi=\omega \text { subject to } \psi=0 \text { on } \Gamma_{2}
$$


in the contained wake region. Since the flow will again be symmetric about the horizontal axis, we only need to solve in the upper half-plane by using an integral equation around the contour $\Gamma_{2}$, as shown in Figure 2, including the dividing streamline, the section of the cylinder past the separation point and the $x$-axis between the cylinder and re-attachment. To do this we define a new function $\Psi$ where

$$
\psi(x, y)=\frac{\omega}{4}\left(x^{2}+y^{2}\right)+\Psi(x, y),
$$

so that $\nabla^{2} \Psi=0$, and $\Psi(x, y)=-\omega\left(x^{2}+y^{2}\right) / 4$ along the contour $\Gamma_{2}$, since $\psi=0$ on $\Gamma_{2}$ (because it is a streamline).

In a parallel method to that for the outer flow, we now solve for this modified potential function using an integral equation approach. Again, once the boundary is known, this is a linear problem. In this region, we choose to solve for $\Phi(x, y)$, part of the analytic function $W(z)=\Phi+i \Psi$. Using Cauchy's integral formula around $\Gamma_{2}$, then taking the imaginary part and modifying the independent variable to be the arclength gives

$$
\Phi(s)=\frac{1}{\pi} \int_{\Gamma_{2}}\left[\frac{\Psi(t)\left(x^{\prime} \Delta x+y^{\prime} \Delta y\right)}{\Delta x^{2}+\Delta y^{2}}+\frac{\Phi(t)\left(y^{\prime} \Delta x-x^{\prime} \Delta y\right)}{\Delta x^{2}+\Delta y^{2}}\right] d t,
$$

where $\Delta x=x(t)-x(s)$ and $\Delta y=y(t)-y(s)$. Noting that $\Psi$ is known everywhere on the boundary, this gives an integral equation for $\Phi$ on the boundary of the vortex region. As above, the singular part of the integrand can be removed, leaving the modified equation

$$
\int_{\Gamma_{2}}\left[\frac{\Delta \Psi\left(x^{\prime} \Delta x+y^{\prime} \Delta y\right)}{\Delta x^{2}+\Delta y^{2}}+\frac{\Delta \Phi\left(y^{\prime} \Delta x-x^{\prime} \Delta y\right)}{\Delta x^{2}+\Delta y^{2}}\right] d t=\epsilon \Phi(s),
$$

where $\Delta \Psi=\Psi(t)-\Psi(s)$ and $\Delta \Phi=\Phi(t)-\Phi(s)$. In this equation, $\epsilon=0$, but in this form the numerical solution exhibits some ill-posedness and so the equation was regularised allowing $\epsilon$ to be non-zero. Values of $\epsilon \approx 10^{-7}$ were found to be sufficient to negate this behaviour and provide smooth, accurate solutions. The method was checked by computing the exact solution for the Poisson equation within both a triangular region and a square region and comparing. Results were found to be accurate to 7 decimal places. Once $\Phi$ is obtained, the velocity, $\Phi^{\prime}(s)$, on the dividing streamline can be obtained using centred finite differences.

2.3. Dynamic conditions On the boundary of the wake region we assume that there is no pressure difference at each point across the dividing streamline. Applying the Bernoulli equation on both sides of the dividing streamline provides the appropriate condition. 
If the flow is horizontal and gravity is acting vertically downward then in nondimensional variables the dynamic conditions approaching the dividing streamline from the two different regions are

$$
\begin{aligned}
& \frac{p(s)}{\rho}+F^{-2} \eta(s)+\frac{1}{2}\left|q_{\mathrm{O}}(s)\right|^{2}=C_{1}, \\
& \frac{p(s)}{\rho}+F^{-2} \eta(s)+\frac{1}{2}\left|q_{\mathrm{I}}(s)\right|^{2}=C_{2}
\end{aligned}
$$

on $y=\eta(s), s_{\text {sep }}<s<s_{\text {reatt }}=s_{L}$, where $s_{\text {sep }}$ and $s_{\text {reatt }}$ are the separation and reattachment points of the dividing streamline, $F=\sqrt{U^{2} / g R}$ is the Froude number, $C_{1}$ and $C_{2}$ are the Bernoulli constants, $p$ and $\rho$ are the pressure and density respectively, and the subscripts $I$ and $O$ refer to the inner and outer flow regions. Equating the pressure across the dividing streamline results in the equation

$$
\left|q_{\mathrm{o}}(s)\right|^{2}-\left|q_{\mathrm{I}}(s)\right|^{2}=K, \quad s_{\text {sep }}<s<s_{L},
$$

where $K$ is a constant that depends on the conditions in the two regions. Noting that the separation and re-attachment points occur at stagnation points of the inner flow, we obtain that the value of $K$ must be $q_{\mathrm{o}}\left(s_{\text {sep }}\right)=q_{\mathrm{o}}\left(s_{\text {reatt }}\right)=\sqrt{K}$. The value of $K$ is not known a priori and must be obtained as part of the solution. Interestingly the Froude number and hence gravity play no role in the solution. It follows that the orientation of the flow makes no difference.

\section{Numerical solution}

The solution procedure is based on an iteration of the unknown points on the dividing streamline. Since the integral equations for the outer and inner flows are both linear once the shape of this streamline is known, we can begin by making a guess for the shape, solve the inner and outer flow problems, and then improve on the guess using Newton's method. It was found that the best way to solve the problem was to fix the total arclength of the dividing streamline after the separation point, $S=s_{L}-s_{\text {sep. }}$. The separation point on the cylinder was left as an output of the iteration scheme.

To solve for the outer flow, all variables are taken to be a function of the arclength from the front of the body, and so we begin by setting

$$
s_{0}=0, \quad s_{1}=\Delta s, \quad s_{2}=2 \Delta s, \quad \ldots, \quad s_{N}=N \Delta s=s_{L},
$$

where $\Delta s$ is the spatial step, $\Delta s=s_{L} / N$. Next, we determine the values of $x_{j}=x\left(s_{j}\right)$ and $y_{j}=y\left(s_{\jmath}\right)$ at $s_{j}, j=1,2, \ldots, I_{\text {sep }}$, on the body corresponding to the surface of the cylinder, and for the values of $y_{j}, j=I_{\text {sep }}+1, \ldots, N$, the location of the dividing 
streamline. Using the known values of $y^{\prime}\left(s_{j}\right), j=1,2, \ldots, I_{\text {sep }}$, on the body and using finite differences (centred) to obtain $y^{\prime}\left(s_{j}\right), j=I_{\text {sep }}+1, \ldots, N$, and Equation (2.1) to obtain $x^{\prime}\left(s_{j}\right), j=1, \ldots, N$, we can integrate from the separation point to find $x_{j}, j=I_{\text {sep }}, \ldots, N$. The shape and slope of the object and wake (given our guess) are now known. Note that once a computation starts, $I_{\text {sep }}$ does not change, so a reasonably accurate starting point is necessary to maintain a consistent interval between the points on the surface of the cylinder and those in the wake. The grids upstream and downstream of the separation point may differ slightly after several iterations as this point moves over the surface of the cylinder.

This information can now be used to find the values of velocity in the $x$-direction, $u_{j}=u\left(s_{j}\right), j=1, \ldots, N$, by solving the integral equation (2.4) in discrete form

$$
\frac{1}{\pi} \sum_{k=0}^{N} \mathscr{K}_{k, j} w_{k} \Delta t_{k}-u_{j}+E_{j}=0, \quad j=1,2, \ldots, N,
$$

where

$$
\begin{aligned}
\mathscr{K}_{k, j}= & \left(u_{k}-u_{j}\right) K_{1}\left(s_{j}, t_{k}\right)+\left(u_{k}-1\right) K_{2}\left(s_{j}, t_{k}\right) \\
& -\left(v_{k}-v_{j}\right) K_{3}\left(s_{j}, t_{k}\right)-v_{k} K_{4}\left(s_{j}, t_{k}\right)
\end{aligned}
$$

and

$$
\begin{aligned}
E_{j}= & 1+\frac{\left[u_{j}-1\right]}{\pi}\left(\arctan \left[\frac{y_{N}-y_{j}}{x_{N}-x_{j}}\right]-\arctan \left[\frac{y_{0}-y_{j}}{x_{0}-x_{j}}\right]\right) \\
& -\frac{v_{j}}{2 \pi} \log \left|\frac{\left(x_{N}-x_{j}\right)^{2}+\left(y_{N}-y_{j}\right)^{2}}{\left(x_{0}-x_{j}\right)^{2}+\left(y_{0}-y_{j}\right)^{2}}\right|,
\end{aligned}
$$

where the weight function $w_{k}$ is chosen depending on the numerical integration scheme being used (in this case the trapezoidal rule) and $\Delta t_{k}$ is the interval size. Note also that from (2.3), $v_{j}=u_{j} x_{j}^{\prime} / y_{j}^{\prime}, j=1,2, \ldots, N$, throughout. This is a set of $N$ linear equations in the $N$ unknowns $u_{\jmath}, j=1,2, \ldots, N$, and is easily solved. Note that $u_{0}=0$ is known since it is the leading stagnation point at the front of the cylinder.

The next step is to solve the inner problem by solving the integral equation (2.5) using collocation in a manner identical to the outer flow. The discrete arclength variable now adopts the points of the outer flow on the dividing streamline and an identical discrete interval around the remainder of $\Gamma_{2}$. This gives a linear set of equations, this time for the values of the function $\Phi_{j}=\Phi\left(s_{j}\right), j=0,1,2, \ldots, M$, now around the inner contour, $\Gamma_{2}$. The velocity of the inner fluid is then obtained as $\Phi^{\prime}\left(s_{j}\right)$.

Using the computed velocities in the inner and outer regions along the dividing streamline it is possible to find the error in the condition (2.6) on the dividing streamline at each point $s_{j}, j=I_{\text {sep }}, \ldots, N-1$, noting that $y\left(s_{N}\right)=0$. Thus there are $N-1-I_{\text {sep }}$ 
equations for the unknown values of $y_{j}, j=I_{\text {sep }}, \ldots, N-1$. However, the separation velocity $\sqrt{K}$ is also an unknown, and so we omit the point $y_{N-1}$ and calculate it at each iteration using interpolation, and replace it as an unknown by $K$.

The error calculated in this way forms the basis of a Newtonian iteration scheme to update the location of the dividing streamline, including the separation point, for our fixed value of vorticity $\omega$ and cavity arclength, $S$. The Jacobian matrix was computed numerically. The separation point $y\left(I_{\text {sep }}\right)$ is actually computed as an angle around the surface of the cylinder, thus allowing it to slide back and forth, even allowing separation from the front half of the cylinder if necessary. At each step, this iteration must include a re-calculation of both the initial grid in terms of arclength and the velocity of the fluid over the updated wake shape and within the trapped flow region.

Solutions with steps of $\Delta s=0.03$ throughout agreed very well with those of $\Delta s=0.06$, and so it appeared the solutions were fully converged at these values.

Despite its rather convoluted nature, this algorithm worked very well and usually converged to a solution within a small number of iterations provided the initial guess (including the location of the separation point) was sufficiently accurate. The zero vorticity solution, with $\omega=0$, was always sufficient as a starting point and in most cases bootstrapping from previous solutions (at lower vorticity, $\omega$ ) was used.

\section{Simulations and results}

A series of simulations was performed for a range of different arclengths and vorticities. For each simulation the value of the arclength was fixed and the vorticity was increased from zero upward until the solutions no longer converged.

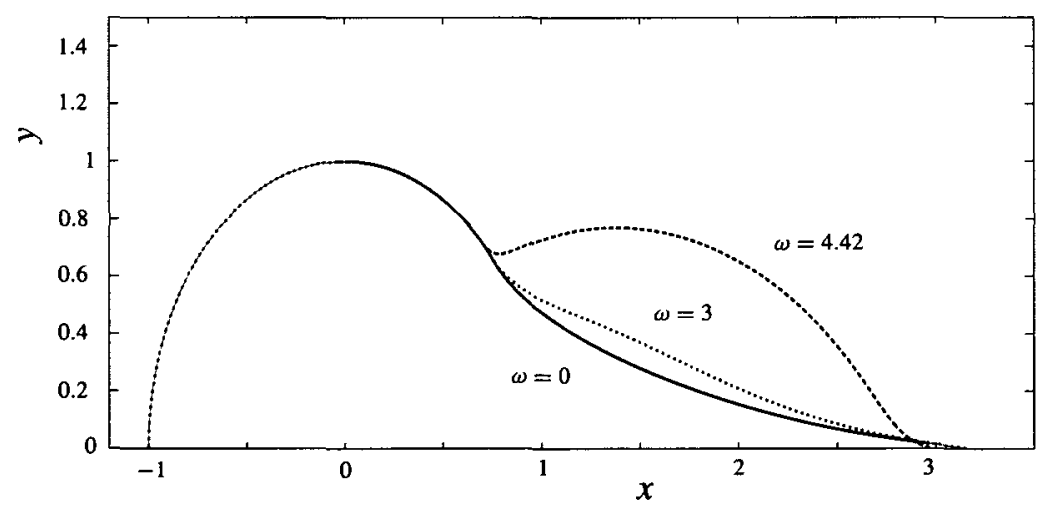

FIGURE 3. The shape of the eddy region for $\omega=0,3,4.42(\max )$ for $S=2.5$.

Typical short wake solutions (arclength $S=2.5$ ) for vorticity values of $\omega=$ $0,3,4.42$ are given in Figure 3. The case $\omega=4.42$ is the value above which 


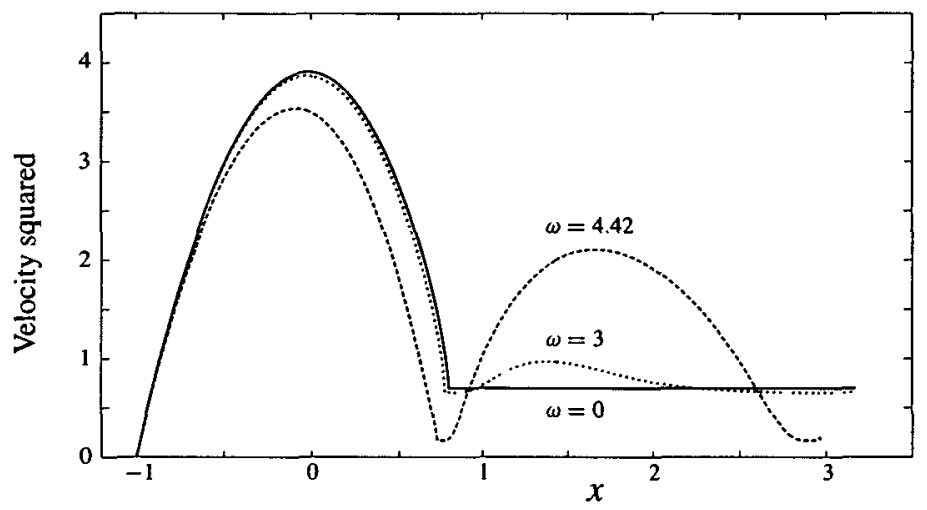

FIGURE 4. Square of separation speed for the outer flow, $\omega=0,3,4.42(\max ), S=2.5$.

no further solutions could be found. Corresponding speeds over the surface of the cylinder and wake are shown in Figure 4. At low values of vorticity, the speed along the dividing vortex sheet is almost constant (as it must be) but varies considerably as the vorticity is increased. The shape of the region varies little at small $\omega$, and even as $\omega$ increases it only fattens slightly. However, as the upper limit of solutions obtained using this method is approached, the eddy region becomes rapidly fatter and the separation point moves up the cylinder and the reattachment point moves toward the back of the cylinder. The limiting case approaches a flow with stagnation points in the outer flow at both separation and reattachment. It was not possible to exactly compute these limiting flows in spite of numerous attempts.

Figure 5 shows the separation speed, $q_{\mathrm{sep}}$, and separation angle as $\omega$ increases from $\omega=0$ up to the maximum value obtained for the arclength $S=2$. Clearly none of these values change significantly until the maximum is approached, when there is a rapid drop in speed and a slight increase in separation angle. This is accompanied by a rapid movement of the reattachment point back toward the rear of the cylinder.

At larger values of the arclength, greater than $S \approx 2$, the solutions no longer appear to be limited by the formation of a stagnation point on the surface of the cylinder, but rather break down with no sign of a rapid decrease in separation velocity, although the eddy region does become more bulbous as the maximal value is approached. It is possible that the flows are approaching a stagnation point flow, but that the numerical scheme was unable to resolve it (although the same resolution was used in all computations). Figure 6 shows the vorticity, separation angle and separation speed of the flow at the maximum vorticity computed for each arclength. Clearly for $S<2.0$ stagnation flows appear to exist, but once above this number the separation velocity rapidly rises to a value close to unity. However, Elcrat et al. [6], computed stagnation point solutions for all trailing lengths, and so it is most likely a limitation of 


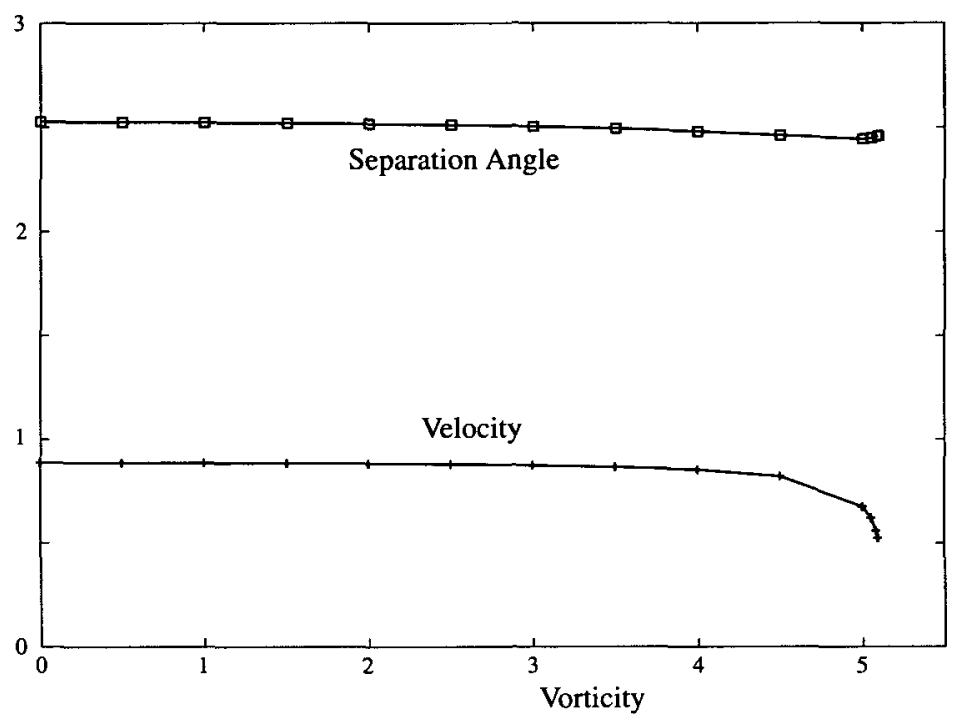

FIGURE 5. Separation angle and separation speed as $\omega$ increases for $S=2$.

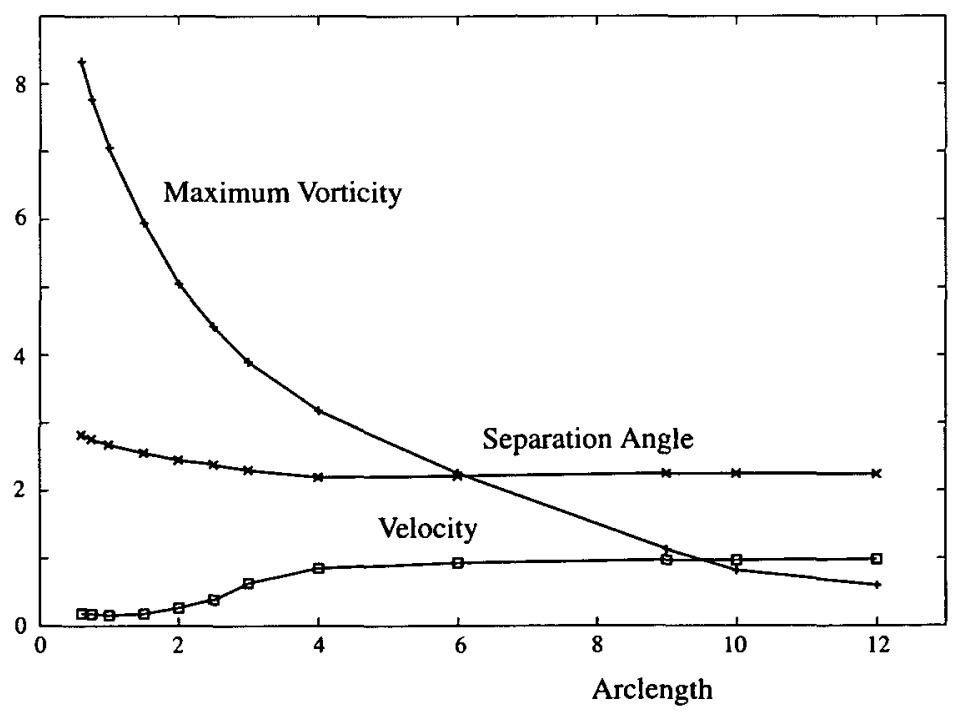

FIGURE 6. Values of vorticity $\omega$, separation angle, $\alpha$, and separation velocity, $q_{\mathrm{sep}}$, for maximum vorticity at which solutions were computed, plotted against cavity arclength, $S$. 
the numerical scheme that these were not obtained in this work. The separation angle decreases as vorticity increases (moving upward on the rear face of the cylinder) and then levelling off as arclength increases. The maximum vorticity, however, appears to continue to decrease as arclength increases. No solutions were found with non-zero vorticity in which the separation occurred on the front side of the cylinder.

\section{Conclusions}

In this paper we have used a matched integral equation approach to compute the steady, high Reynolds number flow around a circular cylinder with a trailing region of uniform vorticity, that is, Prandtl-Batchelor flows. The method is very accurate and the results reproducible. It is shown that at small values of the length of the eddy region a stagnation point appears to form at both separation and re-attachment, and that this event corresponds to the maximum vorticity at which this method will give solutions. At longer lengths, this numerical method was unable to compute such stagnation solutions, but again a maximal vorticity was still found beyond which the method failed to converge. Elcrat et al. [6], however, showed that such solutions do exist, and so it is clear that this is a limitation of the numerical scheme. The limiting vorticity at which solutions could be obtained decreased in magnitude as the length of the trapped vorticity region increased. It is not clear whether the breakdown of solutions is due to the numerical method or has some "physical" cause. It may be that the upper vorticity limit is the point at which multiple vortices form in the wake region or the flow becomes unsteady or it may be simply that the method used herein is not able to calculate solutions for these parameter values. Work is continuing on this problem.

\section{References}

[1] G. K. Batchelor, "On steady laminar flow with closed streamlines at large Reynolds number", J. Fluid Mech. 1 (1956) 177-190.

[2] G. K. Batchelor, "A proposal concerning laminar wakes behind bluff bodies at large Reynolds number", J. Fluid Mech. 1 (1956) 388-398.

[3] G. K. Batchelor, An Introduction to Fluid Dynamics (Cambridge University Press, Cambridge, 1967).

[4] S. Childress, "Solutions of Euler's equations containing finite eddies", Phys. Fluids 9 (1966) 860-872.

[5] E. Cumberbatch and T. Y. Wu, "Cavity flow past a slender hydrofoil", J. Fluid Mech. 11 (1961) 187-208.

[6] A. Elcrat, B. Fornberg, M. Horn and K. Miller, "Some steady vortex flows past a circular cylinder", J. Fluid Mech. 409 (2000) 13-27. 
[7] L. K. Forbes, "On the effects of non-linearity in free-surface flow about a submerged point vortex", J. Eng. Maths 19 (1985) 139-155.

[8] B. Fornberg, "Steady viscous flow past a circular cylinder up to Reynold's number 600 ", J. Comp. Physics 61 (1985) 297-320.

[9] B. Fornberg, "Steady incompressible flow past a row of circular cylinders", J. Fluid Mech. 225 (1991) 655-671.

[10] M. Gurevich, Theory of jets and ideal fluids (Academic Press, New York, 1955).

[11] H. Lamb, Hydrodynamics, 6th ed. (Cambridge University Press, Cambridge, 1932).

[12] J. A. Liggett and P.-F. Liu, The boundary element method for porous media flow (Allen and Unwin, London, 1983).

[13] M. J. Lighthill, “A note on cusped cavities”, Tech. Rep. 2328, Aero. Res. Coun. Rep. Mem., 1946.

[14] M. J. Lighthill, "On boundary layers and upstream influence, I. A comparison between subsonic and supersonic flows", Proc. Roy. Soc. Lond. A 217 (1953) 344-357.

[15] D. Moore, P. Saffman and S. Tanveer, "The calculation of some Batchelor flows: The Sadovskii vortex and rotational corner flow", Phys. Fluids 31 (1988) 978-990.

[16] V. S. Sadovskii, "Vortex regions in a potential stream with a jump of Bernoulli's constant at the boundary", Appl. Math. Mech. 35 (1971) 729.

[17] F. T. Smith, "A structure for laminar flow past a bluff body at high Reynolds number", J. Fluid Mech. 155 (1985) 175-191.

[18] R. V. Southwell and G. Vaisey, "Fluid motions characterised by 'free' streamlines", Phil. Trans. Roy. Soc. A 240 (1946) 117-161.

[19] J.-M. Vanden-Broeck, "Nonlinear capillary free-surface flows", J. Eng. Math. 50 (2004) 415-426, Lighthill Memorial Paper.

[20] J.-M. Vanden-Broeck and E. O. Tuck, "Steady inviscid rotational flows with free surfaces", J. Fluid Mech. 258 (1994) 105-113. 